\title{
Influência dos elementos meteorológicos na eficiência da geração de energia elétrica em células fotovoltaicas
}

\author{
Elisardo Porto ${ }^{1}$ and Cássio Suski ${ }^{1}$
}

\begin{abstract}
Resumo
A produção de energia limpa tem sido uma das principais buscas globais nos últimos anos. As faixas de radiação, enviadas através da luz solar, que podem ser captadas por células fotovoltaicas, que transformam a luz em energia elétrica, tornam o sol na fonte mais promissora para as necessidades elétricas dos seres humanos. $\mathrm{O}$ clima tem uma considerável importância na captação de energia solar, pois a eficiência de um sistema de células érelacionado diretamente com as condições climáticas locais. O objetivo deste artigo é estabelecer uma relação entre geração de energia elétrica por meio de células fotovoltaicas e os elementos meteorológicos, de poluição, radiação e temperatura das células fotovoltaicas, a fim de definir as melhores condições para ampliação da eficiência das células, bem como proporcionar a redução da emissão de gases de efeito estufa. A metodologia proporciona a mensuração da geração de energia elétrica, a compilação dos elementos meteorológicos, $\mathrm{CO}_{2}$, UVA, UVB e temperatura de placa, a relação entre a geração de energia elétrica e os demais parâmetros e a criação de cenários para análise da emissão de gases de efeito estufa. Os resultados esperados são o estabelecimento de uma relação entre a geração de energia elétrica por meio de células fotovoltaicas e os parâmetros, bem como possibilitar a redução da emissão de gases de efeito estufa por meio da ampliação do uso dessa fonte e redução da geração de energia por hidrelétricas e termelétricas.
\end{abstract}

\section{Palavras-chave}

fotovoltaica, meteorológicos, GEE.

\section{Introdução}

O aquecimento global e a produção de energia limpa são importantes preocupações relacionadas ao clima e ao meio ambiente. A falta de investimentos aliado à falta de informações e de projetos práticos por parte do poder público justifica o lento avanço nas conquistas de energias limpas.

$\mathrm{O}$ aumento da temperatura global é potencializado pelas ações antrópicas que interferem no efeito estufa natural do planeta devido ao aumento de emissões de gases de efeito estufa, oriundos da queima de combustíveis fósseis de usinas termelétricas, da decomposição da matéria orgânica que sobra do corte das árvores e do carbono presente no solo alagado pelas hidrelétricas, dentre outras fontes emissoras.

O aproveitamento da energia gerada pelo sol, inesgotável na escala terrestre de tempo, tanto como fonte de calor quanto de luz, é hoje uma das alternativas energéticas mais promissoras para prover a energia necessária ao desenvolvimento humano. Quando se fala em energia, devese lembrar de que o sol é responsável pela origem de praticamente todas as outras fontes de energia na Terra (PINHO; GALDINO, 2014).

Os fatores atmosféricos geralmente observados na produção de energia solar são a nebulosidade, umidade do ar e temperatura ambiente, mas a disponibilidade de radiação solar, que é a energia incidente sobre a superfície terrestre, vai ser influenciada pela inclinação da terra em relação ao sol que varia durante o decorrer do dia, do mês e do ano (ANEEL, 2008).
A duração da luz solar varia conforme a região doplaneta e períodos do ano, especificamente cita-se a questão dos solstícios que ocorrem no polo norte, quando em 21 de dezembro pode chegar até a não existir exposição solar, ou em 21 de junho, quando pode chegar a até 24 horas de exposição solar. Os primeiros estudos sobre o efeito Fotovoltaico foram observados 1839 e aprimorados com uso de outros materiais, mas de maneira arcaica em 1877. Os primeiros grandes avanços nesses estudos ocorreram no período entre os anos 1930 e 1950 do século passado, com a utilização das células fotovoltaicas compostas por cristaisde silício que são a base das células atuais (VALLERA; BRITO, 2006).

O efeito fotoelétrico é um processo físico do elemento que converte luz solar em energia elétrica, sendo que a energia da luz absorvida pela célula fotovoltaica é transferida a seus átomos e elétrons e estes elétrons desprendem-se dos átomos componentes do material semicondutor, a corrente elétrica através do fluxo elétrico ordenado (Ghensev, 2006).

Os módulos fotovoltaicos são o conjunto de células fotovoltaicas encapsuladas, cujo objetivo desse empacotamento

\footnotetext{
${ }^{1}$ Mestrado em Clima e Ambiente, Instituto Federal de Santa Catarina Email: cassio.suski@ifsc.edu.br (Cássio Suski)

Corresponding author:

Elisardo Porto, Mestrado em Clima e Ambiente, Instituto Federal de Santa Catarina

Email: elisardo.porto@gmail.com
} 
é para que as células fiquem protegidas das intempéries, principalmente da umidade do ar (Fadigas, Energia, \& Paulo, 2013).

A geração fotovoltaica pura é aquela que não possui outra forma de geração de eletricidade. Devido ao fato do sistema só gerar eletricidade nas horas de sol, os sistemas autônomos ou off-grid são dotados de acumuladores que armazenam a energia para os períodos sem sol, o que acontece todas as noites, e também nos períodos chuvosos ou nublados. Os acumuladores são baterias dimensionadas de acordo com a autonomia do sistema. O Sistemas offgrid da rede elétrica convencional para carregamento de bancos de baterias, necessita de um controlador de carga com tecnologia MPPT (Maximum Power Point Tracking ou Rastreamento do Ponto de Máxima Potência).

As condições climáticas são importantes na geração de energia solar, pois o excesso ou falta de alguns fenômenos pode afetar a captação de energia. Um clima com umidade alta e sem vento pode gerar condições de calor excessivo junto às células, o que prejudica a produção energética e, além disso, a umidade pode gerar precipitação e nuvens que encobrem e dificultam a absorção da radiação solar. O vento é um dos elementos primordiais, pois ele movimenta as massas de ar, empurrando a umidade, as nuvens eresfriando o sistema fotovoltaico.

O presente estudo pretende analisar a influência dos elementos meteorológicos na eficiência da geração de energia elétrica por meio de células fotovoltaicas e para isso será discorrido, dentre outros assuntos, sobre as principais fontes de geração de energia elétrica, as respectivas emissões de gases de efeito estufa e a influência dos elementos meteorológicos na geração de energia por células fotovoltaicas.

\section{Metodologia}

Para o desenvolvimento da pesquisa e análise da influência dos elementos meteorológicos na eficiência da geração de energia elétrica por meio de células fotovoltaicas o estudo foi dividido em 4 etapas:

- Etapa 1: Mensuração da geração de energia elétrica por meio de células fotovoltaicas;

- Etapa 2: Compilação dos elementos meteorológicos, $\mathrm{CO}_{2}$, UVA, UVB e temperatura de placa;

- Etapa 3: Relação entre a geração de energia elétrica por meio de células fotovoltaicas e os demais parâmetros;

- Etapa 4: Parâmetros para melhoria da eficiência da geração de energia limpa;

\section{Mensuração da geração de energia elétrica por meio de células fotovoltaicas}

A mensuração da taxa de geração de energia elétrica local está prevista para o período de novembro de 2021 a março de 2022.

Será utilizado um Kit Gerador de Energia Solar com células fotovoltaicas marca Canadian Solar, modelo CS6U325P (Figura 1), acoplado à Estação Meteorológica protótipo de Sanches (2020) (Figura 2), com sensores de direção do vento (sensor HMC-5883), velocidade do vento (sensorHall - US-1881), umidade relativa do ar (sensor HDC-1080), pressão atmosférica e temperatura (BMP-180), bem como sensores de UVA, UVB ML8511, Sensor de Temperatura Termopar Tipo K da célula solar e Sensor Dióxido de Carbono $\mathrm{CO}_{2}$ NDIR MH-Z14A e transmissão dos dados realizada via cabo de comunicação RS485 para Usb com software de leitura instalado.

O conjunto, constituído por estruturas mecânicas e eletroeletrônicas, será instalado em área livre, sobre o telhado do bloco 2 do IFSC, Câmpus Itajaí, a aproximadamente $12 \mathrm{~m}$ de altura em relação ao solo (figura 3), nas coordenadas Lat: $26^{\circ} 55^{\prime} 51^{\prime \prime} \mathrm{S}$, Lon: $048^{\circ} 41^{\prime} 05^{\prime \prime}$ $\mathrm{O}$.

O local da instalação foi definido de modo que evite interferências de outras edificações nos elementos meteorológicos envolvidos nas análises.

A estrutura mecânica será constituída de um suporte metálico para fixação das células fotovoltaicas e encaixe dos sensores e da estação meteorológica.

A estrutura eletroeletrônica será composta por um controlador XTRA4210N-XDS2 de carga e descarga de baterias e corrente de passagem de 40A com tecnologia MPPT (Maximum Power Point Tracking, ou Rastreamento do Ponto de Máxima Potência), sendo que o referido equipamento estará instalado no Laboratório de Eletroeletrônica, situado no Bloco 2, segundo piso, no referido Campus, juntamente com o banco de 03 baterias Modelo 12MN105, o módulo inversor Modelo IP2000-22 e o sistema de proteção com disjuntores, dispositivos de proteção de aterramento e pára-raios.

A Figura 4 mostra o esquema de disposição formado pelo sistema de células fotovoltaicas, estação meteorológica e dispositivos de proteção.

O conjunto de geração de energia será instalado e, posteriormente, calibrado por meio de conferência com multimetro (ET-1002 Minipa) na entrada e saída do controlador, a fim de verificar se a tensão $(37,0 \mathrm{~V})$ e corrente $(8,78 \mathrm{~A})$ estão adequadas e, consequentemente, a potência a ser gerada $(325 \mathrm{~W})$.

O sistema operará off grid (onde o sistema não está ligado à rede da distribuidora oficial de energia), e estará ligado a um equipamento de consumo de carga (ventilador), durante todo o período de geração de energia. Este é um fator essencial para o correto funcionamento do controlador do sistema das placas fotovoltaicas, pois o mesmo interrompe seu funcionamento em caso de acúmulo de carga máxima pelo banco de baterias. Após a carga limite de armazenamento o controlador interrompe a passagem de corrente e tensão para as baterias, mesmo mantendo a passagem de energia para o ventilador, porém esta alternância de corrente interrompe a leitura dos parâmetros objetos do estudo.

A leitura da tensão gerada (V), corrente produzida (A) e potência calculada (W) será realizada, a cada 15 minutos, por meio de controlador de carga XTRA4210N-XDS2 que disponibiliza em tempo real em display e em software LandStar B(LS-B). Esses dados serão disponibilizados em forma de tabelas e gráficos a fim de permitir a análise da geração da energia elétrica. 


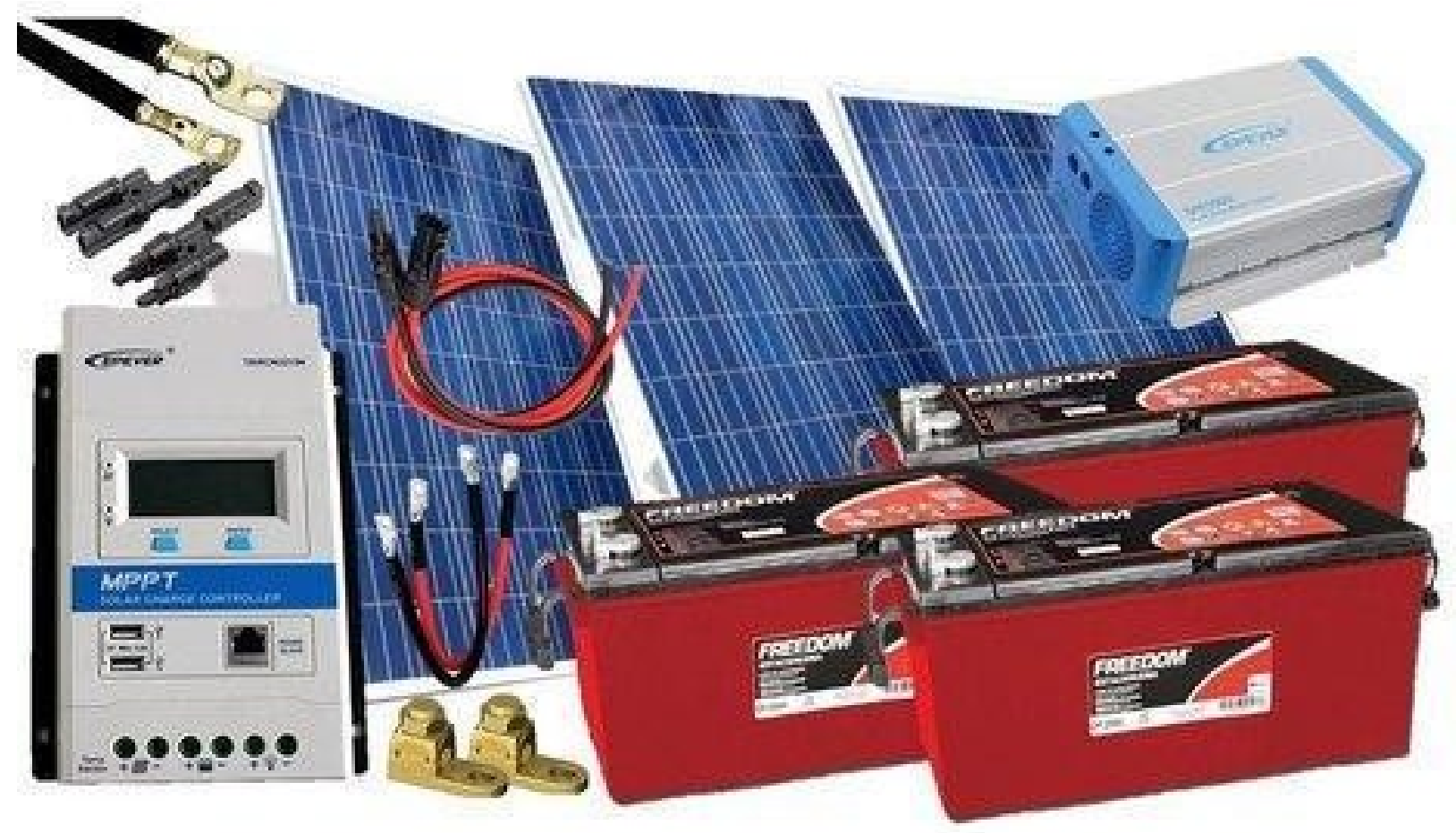

Figure 1. Kit Gerador de Energia Solar.

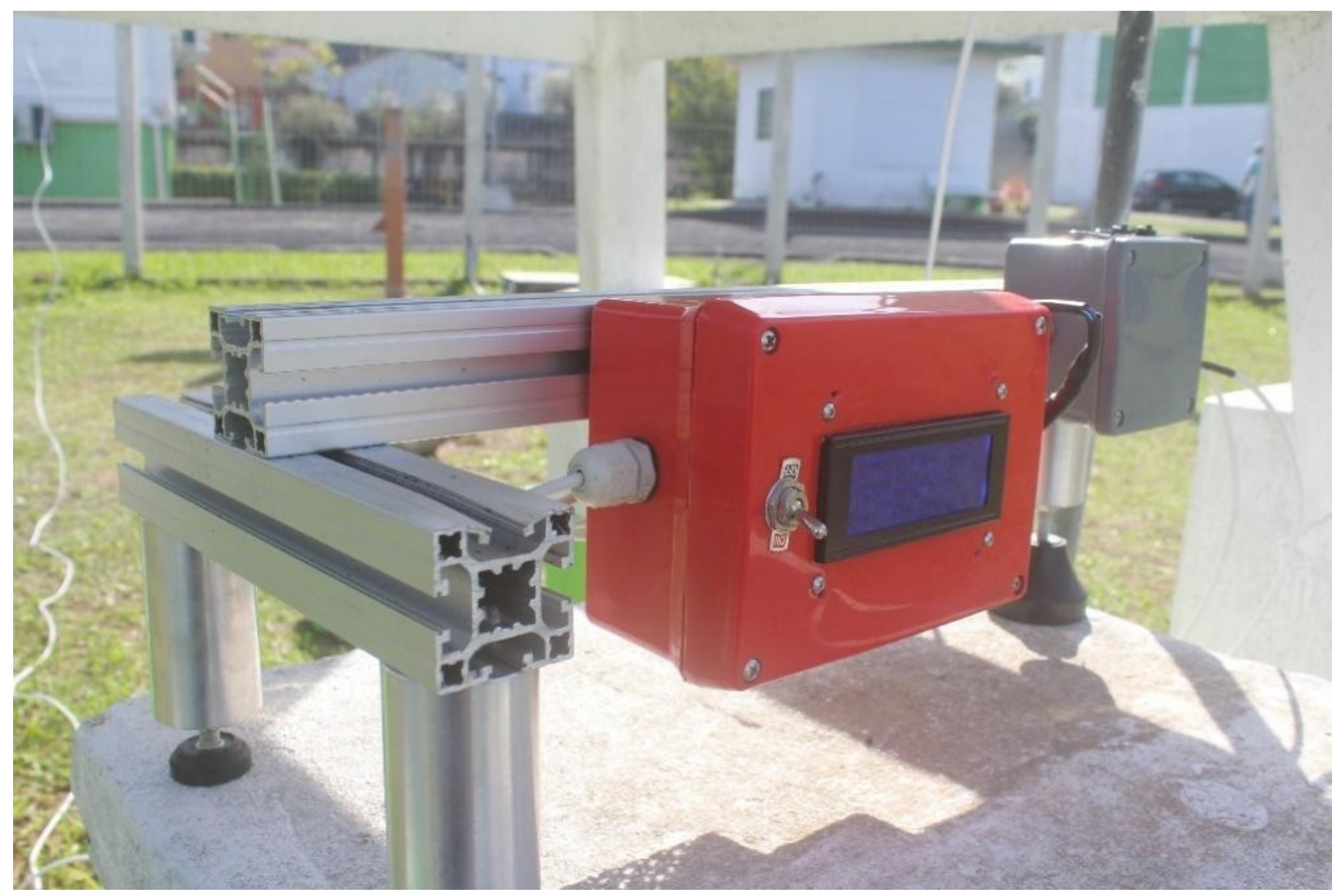

Figure 2. Estação Meteorológica 


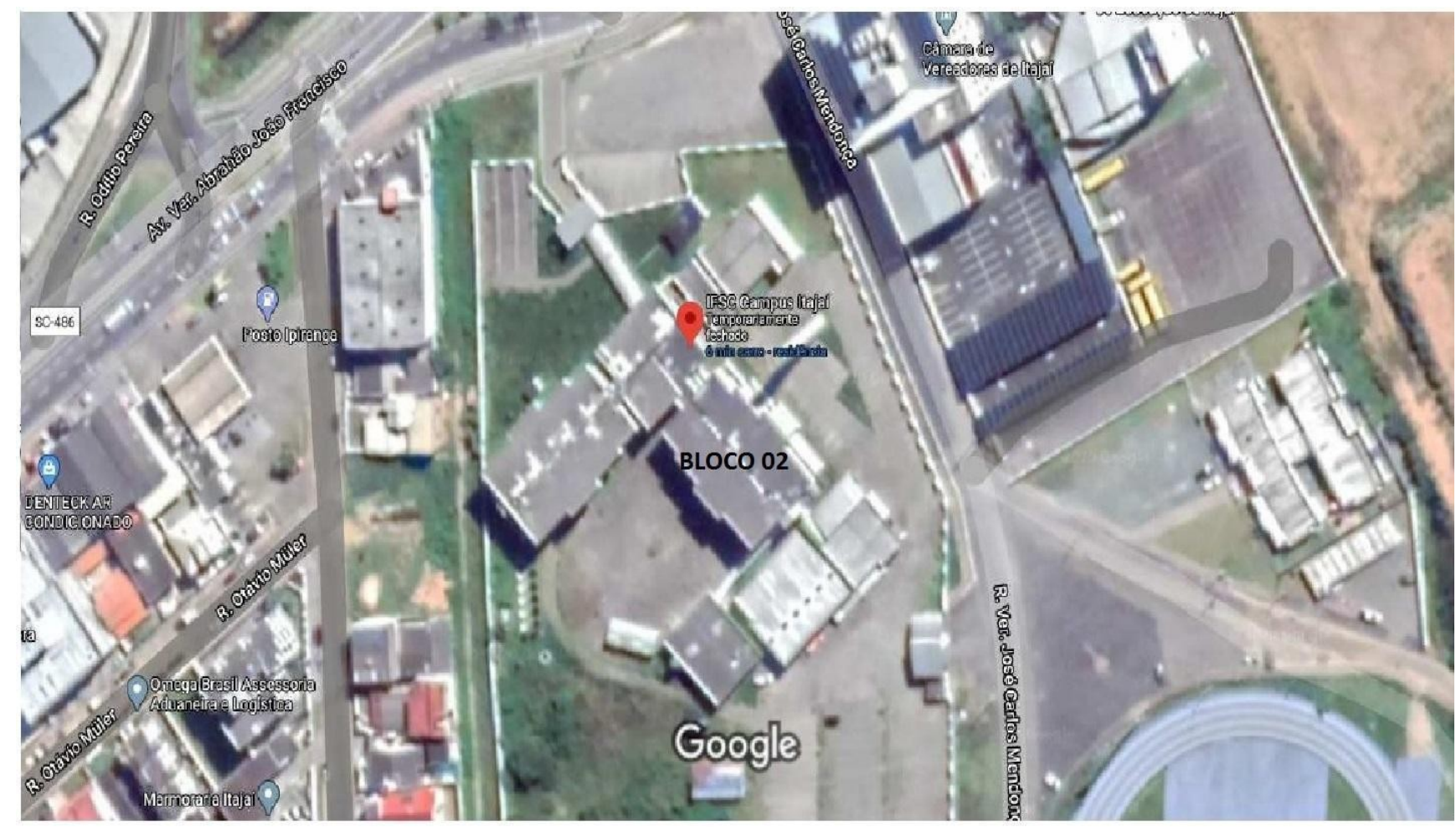

Imagens @2020 CNES / Airbus, Maxar Technologies, Dadas do mapa @2020 $20 \mathrm{~m}$

Figure 3. Imagem satélite IFSC Câmpus Itajaí - SC

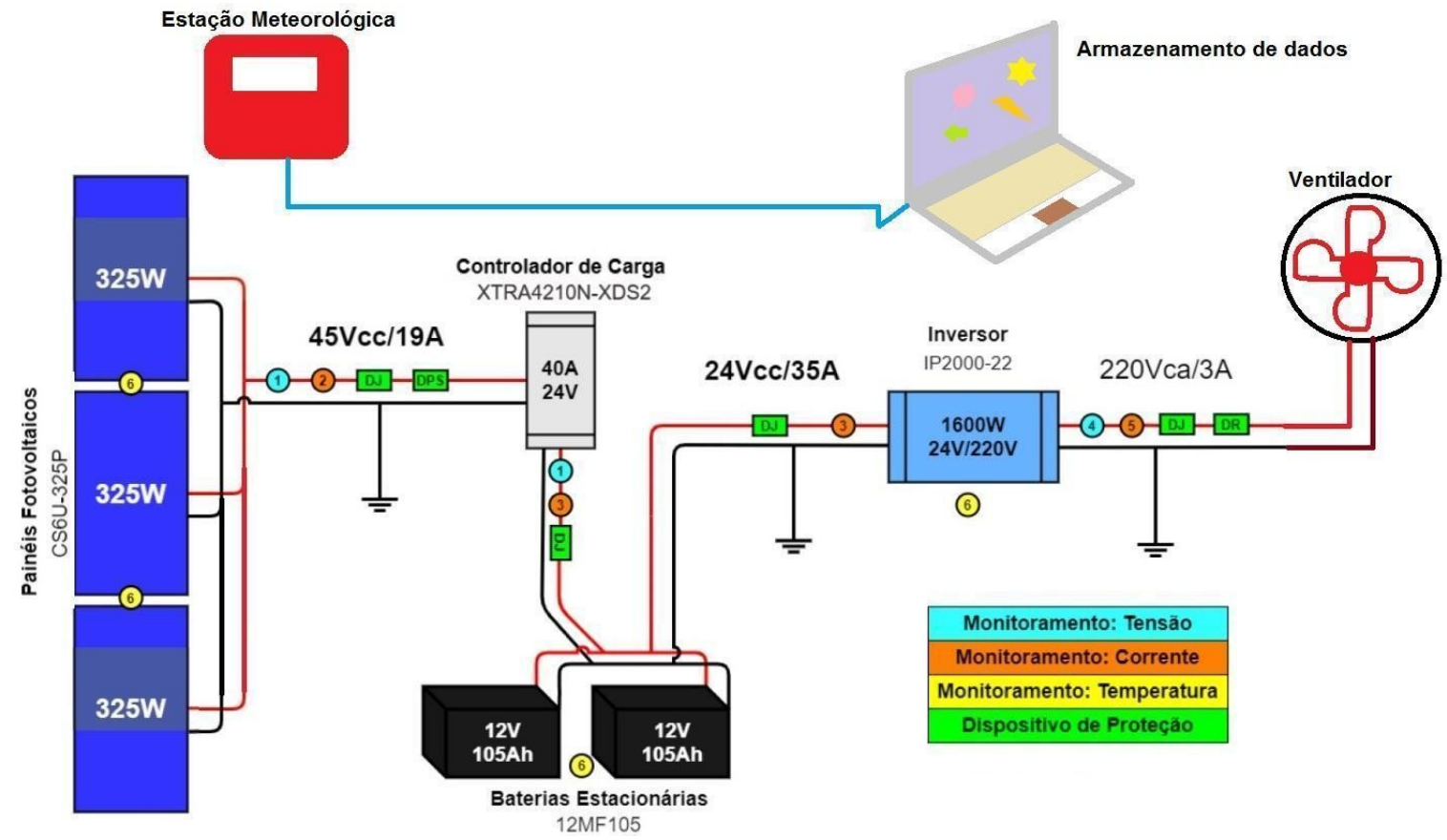

Figure 4. Esquema de instalação de sensores 
Posteriormente, os dados de leitura da tensão, corrente e potência serão relacionados com os elementos meteorológicos, de poluição, UVA, UVB e temperatura da placa.

\section{Compilação dos elementos meteorológicos, $\mathrm{CO}_{2}$, UVA, UVB e temperatura de placa}

A estação meteorológica realizará a leitura dos elementos meteorológicos velocidade e direção do vento, umidade relativa do ar, pressão atmosférica, temperatura do ar, $\mathrm{CO}_{2}$, radiação UVA e UVB e temperatura da placa, de maneira sincronizada com a leitura do controlador de carga (energia elétrica) a cada 15 minutos.

A estação meteorológica utilizará uma plataforma de prototipagem eletrônica de hardware livre e de placa única (Arduino) que utiliza a linguagem de programação $\mathrm{C}++$. Tal plataforma gerará um banco de dados dos parâmetros acima mencionados.

\section{Relação entre a geração de energia elétrica por meio de células fotovoltaicas e os demais parâmetros}

Após a mensuração e compilação dos dados obtidos nas linguagens de programação C++ e LS-B (Embarcado), formatar-se-á a tabela 1 , bem como gráficos de correlação para discussão e análise da influência dos elementos meteorológicos na geração de energia.

Nesta etapa serão relacionados os parâmetros de geração de energia elétrica (Tensão, corrente e potência) com os parâmetros meteorológicos (Velocidade e direção do vento, umidade relativa do ar, pressão atmosférica, temperatura), de poluição $\left(\mathrm{CO}_{2}\right)$, radiação (UVA e UVB) e temperatura da placa na eficiência da geração de energia elétrica das células fotovoltaicas.

Tabela 1: Relação entre a geração de energia elétrica por meio de células fotovoltaicas e os demaisparâmetros

\section{Parâmetros para melhoria da eficiência da geração de energia limpa}

Por meio da interpolação dos parâmetros poderão ser geradas hipóteses de uso em relação a posição e condições de montagem e eficiência das células fotovoltaicas, proporcionando a redução da utilização de energia elétrica gerada por hidroelétricas e termoelétricas e, consequentemente, prever a influência destes no impacto ambiental e na geração de gases de efeito estufa (GEE).

$O$ efeito da melhoria da eficiência da geração de energia limpa no ambiente será medido por meio de um levantamento da possível redução da área coberta pelos lagos das usinas hidroelétricas e pela redução do consumo de combustíveis (fósseis, carvão e bagaço de cana, etc) nas termelétricas e, consequentemente, da emissão de GEE.

A tabela 2 mostra a capacidade produzida por metro quadrado de lago, em algumas hidrelétricas do Brasil.

Para estimativa de emissão de $\mathrm{CO}_{2}$ na geração de energia hidroelétrica foi considerado o fator de emissão de 86,21 $\mathrm{gCO}_{2} / \mathrm{KWh}$ (ou ton/GWh), estimado por Miranda (2012) considerando estudos de avaliação do ciclo de vida que englobasse etapas de construção e geração e emissões de reservatórios, uma vez que estes possuem emissões significativas e caracterizam a fase de operação da usina.

A tabela 3 mostra os cenários estabelecidos para avaliação da redução da emissão de GEE. Baseado nos cenários propostos será calculada a redução de emissão de GEE baseado no o fator de emissão de Miranda (2012) e a consequente influência na variação da temperatura por meio da equação 1 (Ellis, 2013).

$$
\begin{aligned}
& \Delta T=1,66 \ln (C / C o)(1) \\
& \text { Onde: } \Delta \mathrm{T}=\text { Variação da temperatura }\left({ }^{\circ} \mathrm{C}\right) \\
& \mathrm{C}=\text { Emissão de } \mathrm{CO}_{2} \text { final }(\mathrm{ppm}) \\
& \mathrm{C}_{0}=\text { Emissão de } \mathrm{CO}_{2} \text { inicial }(\mathrm{ppm})
\end{aligned}
$$

\section{Resultados e Discussão}

Será estabelecida uma relação entre a geração de energia elétrica por meio de células fotovoltaicas e os elementos meteorológicos (velocidade e direção do vento, umidade relativa do ar, pressão atmosférica e temperatura do ar), de poluição $\left(\mathrm{CO}_{2}\right)$, radiação (UVA e UVB) e temperatura das células fotovoltaicas, a fim de definir as melhores condições para ampliação da eficiência de geração de energia elétrica.

Pretende-se ainda possibilitar a redução da emissão de gases de efeito estufa por meio da ampliação da geração de energia elétrica com células fotovoltaicas e redução da geração de energia por hidrelétricas e termelétricas e, consequentemente, proporcionar condições para redução da temperatura atmosférica gerada pelo efeito estufa.

Além disso, a melhoria na eficiência proporcionará a redução da necessidade de fabricação de novos módulos de células fotovoltaicas e, consequentemente, reduzirá a necessidade de utilização de matérias-primas tais como, silício monocristalino e policristalino, boro, fósforo, prata, alumínio, vidro temperado de alta transparência, fluoreto de polivinila e acetato de etilvinila e evitará o descarte de novos conjuntos de células fotovoltaicas.

Demonstrar-se-á a potência gerada durante a vida útil do kit gerador com uma produção normal e dessa forma, ao se obter um índice de melhoria na eficiência poderá se projetar o ganho da geração de energia e calcular a redução da produção de novas placas de células fotovoltaicas.

\section{Conclusões}

As metas traçadas para analisar a influência dos elementos meteorológicos na eficiência da geração de energia elétrica por meio de células fotovoltaicas tornam este trabalho atual e relevante para a incorporação da ideia de preservação ambiental com a geração de energia limpa através do sol.

O levantamento dos dados referentes a temperatura de placa em relação ao elementos climáticos leva a desenvolver novas técnicas e mesmo abrir portas para novas tecnologias empregadas na produção de células fotovoltaicas.

A relevância do estudo dos gases do efeito estufa deve demonstrar que a geração de energia limpa será importante para todos os usuários neste planeta.

A determinação coerente do uso das matérias primas empregadas nos equipamentos e nas indústrias geradoras possibilitará a redução da emissão de dióxido de carbono como apresentado em cenários distintos neste trabalho. 
Table 1. Relação entre a geração de energia elétrica por meio de células fotovoltaicas e os demais parâmetros

\begin{tabular}{|c|c|c|c|c|c|c|c|c|c|c|c|c|c|}
\hline $\begin{array}{l}\text { DT } \\
\mathrm{d} / \mathrm{m} / \mathrm{a}\end{array}$ & $\begin{array}{l}\mathbf{H} \\
\mathrm{h}: \mathrm{m}: \mathrm{s}\end{array}$ & $\begin{array}{l}\text { TA } \\
{ }^{\circ} \mathrm{C}\end{array}$ & $\begin{array}{l}\text { TC } \\
{ }^{\circ} \mathrm{C}\end{array}$ & $\begin{array}{l}T \\
V\end{array}$ & $\begin{array}{l}\text { C } \\
\text { A }\end{array}$ & $\begin{array}{l}\mathbf{P} \\
\mathrm{W}\end{array}$ & $\begin{array}{l}\mathbf{V V} \\
\mathrm{m} / \mathrm{s}\end{array}$ & $\begin{array}{l}\text { DV } \\
\text { o }\end{array}$ & $\begin{array}{l}\text { PA } \\
\mathrm{mmHg}\end{array}$ & $\begin{array}{l}\text { UR } \\
\%\end{array}$ & $\begin{array}{l}\mathrm{CO}_{2} \\
\mathrm{ppm}\end{array}$ & $\begin{array}{l}\text { UVA } \\
\mathrm{mW} / \mathrm{cm}^{2}\end{array}$ & $\begin{array}{l}\text { UVB } \\
\mathrm{mW} / \mathrm{cm}^{2}\end{array}$ \\
\hline
\end{tabular}

Table 2. Capacidade de produção em relação a área inundada.

\begin{tabular}{|c|c|c|c|}
\hline Usina & $\begin{array}{l}\text { Área } \\
\text { inundada } \\
\left(\mathrm{km}^{2}\right)\end{array}$ & $\begin{array}{l}\text { Capacidade } \\
\text { instalada }(\mathrm{MW})\end{array}$ & $\mathrm{MW} / \mathrm{km}^{2}$ \\
\hline Itaipu & 1.350 & 14.000 & 10,37 \\
\hline $\begin{array}{l}\text { Tucuruí } \\
\text { (PA) }\end{array}$ & 2.430 & 8.370 & 3,44 \\
\hline $\begin{array}{l}\text { Porto } \\
\text { Primavera } \\
\text { (SP) }\end{array}$ & 2.250 & 1.540 & 0,68 \\
\hline $\begin{array}{l}\text { Sobradinho } \\
\text { (BA) }\end{array}$ & 4.214 & 1.050 & 0,25 \\
\hline
\end{tabular}

Fonte: Itaipú. Disponível em: https://www.itaipu.gov.br.

\section{References}

Atlas de energia elétrica do brasil / agência nacional de energia elétrica. (2008). Brasília: ANEEL.

Ellis, R. (2013).

Epe, E., Pesquisa, D., \& Energética. (2018).

Fadigas, E. A. F. A., Energia, S. P. D., \& Paulo. (2013).

Ghensev, A. (2006). Monografia do curso de pós-graduação latu sensu em fontes alternativas de energia. UFLA, Lavras.

(n.d.). Retrieved from https: / / www. itaipu.gov.br . Acessoem: 05 denovembrode2 020

Miranda, M. M. (2012).

Pinho, J. T., \& Galdinho, M. A. (2014).

Sanches, S. R. (n.d.).

Vallera, A. M., Brito, M. C., \& Física, G. D. (2006). Meio Século de História Fotovtaica, 29(2), 10-15. 


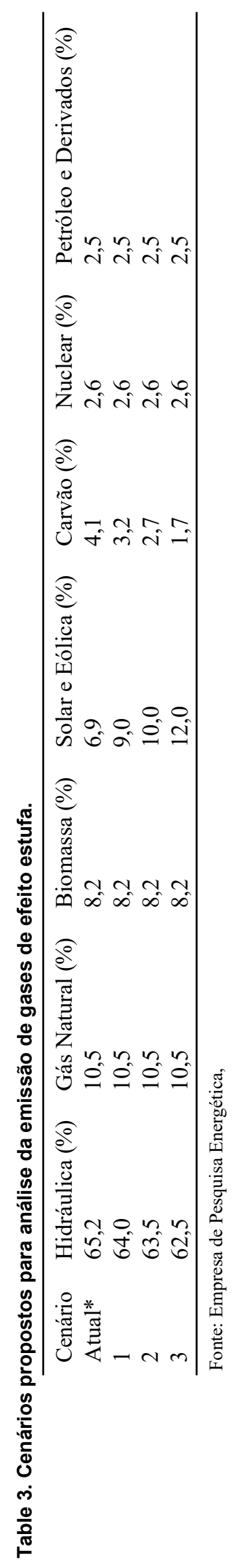

